\title{
The value of an initial drop in Human Chorionic Gonadotropin levels in predicting a response to methotrexate in women with low-risk Gestational Trophoblastic Neoplasia
}

\author{
Paulina Banach ${ }^{1}$, Mikolaj Piotr Zaborowski ${ }^{1}$, Natalia Izycka ${ }^{1}$, \\ Anna Romala², Ewa Nowak-Markwitz ${ }^{1}$ \\ ${ }^{1}$ Department of Gynecology, Obstetrics and Gynecologic Oncology, Division of Gynecologic Oncology, \\ Poznan University of Medical Sciences, Poznan, Poland \\ ${ }^{2}$ Division of Perinatology and Women's Diseases, Poznan University of Medical Sciences, Poznan, Poland
}

\begin{abstract}
Objectives: The early identification of patients who are being treated for low-risk gestational trophoblastic neoplasia (LRGTN) with single-agent chemotherapy, who are at high risk of developing chemoresistance, is of crucial importance. The aim of our research was to evaluate the pretreatment beta subunit of human chorionic gonadotropin ( $\beta \mathrm{hCG}$ ) concentration and its decrease after the administration of the first course of methotrexate (MTX) in predicting later chemo-resistance to single-agent chemotherapy.

Material and methods: A total of 46 patients diagnosed with LRGTN treated with a 5-day methotrexate (MTX) regimen were retrospectively studied. 24 of the patients were successfully cured with only MTX therapy (MTX group). The disease was considered resistant in the remaining 22 patients who, after MTX therapy, required further chemotherapy with an EMA/CO regimen (EMA/CO group). To compare changes in the $\beta \mathrm{hCG}$ concentrations between the two courses of treatment (and the two groups), we calculated the percentage of decline. We determined the specificity and sensitivity of the initial $\beta \mathrm{hCG}$ level and its percentage decline, as a potential predictor of the need for a future EMA/CO regimen. For diagnostic purposes, $\beta$ hCG levels were measured before the first and second administrations of MTX with a commercial ELISA kit.

Results: In the EMA/CO group, we found the initial $\beta$ hCG level before the first MTX dose was higher (median $=6275 \mathrm{mlU} / \mathrm{mL}$, range: $21.53-192.610 .0 \mathrm{mlU} / \mathrm{mL}$ ) than in the $\mathrm{MTX}$ group (median $=532 \mathrm{mlU} / \mathrm{mL}$, range: $56.5 \mathrm{mlU} / \mathrm{mL}-360.397 .0 \mathrm{mlU} / \mathrm{mL}$ ) ( $p=0.034$, Mann-Whitney test). The percentage decreases in the $\beta \mathrm{hCG}$ values relative to the initial concentrations were higher in the MTX group (median decrease $=82.7 \%$, range: from $13.3 \%$ to $99.9 \%$ ) than in the EMA/CO group (median decrease $=71.1 \%$, range: from an increase of $56.1 \%$ to a decrease of $97.1 \%)(p=0.0079$, Mann-Whitney test). An analysis of the ROC curves implied optimal cutoff values for the initial $\beta H C G(6054 \mathrm{IU}$, sensitivity $=55 \%$, specificity $=86 \%)$ and the percentage change in $\beta$ hCG levels (decrease by $76.5 \%$, sensitivity $=72 \%$, specificity $=71 \%$ ).

Conclusions:

1. Women with initially higher $\beta$ hCG levels have a greater risk of developing MTX chemo resistance.

2. It would be advantageous to consider administering an EMA/CO regimen in women with LRGTN when their initial BhCG levels are greater than 6000 .

Key words: methotrexate; predictive values of $\beta$ hCG; gestational trophoblastic neoplasms
\end{abstract}

Ginekologia Polska 2019; 90, 3: 141-147

\section{INTRODUCTION}

Gestational trophoblastic neoplasm (GTN) is a highly curable group of malignant pathologies that develop from trophoblastic cells [1]. It includes placental site trophoblastic tumors (PSTT), invasive moles, epithelial trophoblastic tumors (ETT) and choriocarcinomas. In recent years, one of the big challenges has been to find biomarkers for gestational trophoblastic disease (GTD) that could significantly improve the 
identification of patients who have a higher risk of developing more aggressive forms of the disease. Based on WHO criteria that consider epidemiological data, the course of disease has been qualified as either low-risk (LRGTN) or high-risk (HRGTN). Based on this same WHO risk-factor scoring system as modified by FIGO, patients diagnosed with GTN are classified as having either a low-risk or a high-risk of developing methotrexate (MTX) resistance. The FIGO Prognostic Scoring System is also designed to identify patients with a high risk of developing aggressive GTN [2]. Clinical features included in this scoring system are pretreatment beta subunit of human chorionic gonadotropin ( $\beta \mathrm{hCG}$ ) levels, form of preceding pregnancy, time since the last pregnancy, age, size of the tumor, site of the spread, number of metastases, and the drugs used that failed to treat the tumor. Due to varying dynamics of the disease, this staging system does not always work perfectly in practice. Whereas low-risk GTN patients (who have received $<5$ points) are treated with single-agent chemotherapy, and for high-risk patients (who have received $>=7$ vpoints) multi-agent chemotherapy is recommended. $\beta$ hCG, though included in the WHO/FIGO criteria, is not the only decisive factor in determining future management. In practice, it may happen that patients with a relatively high $\beta$ hCG levels are still qualified as have a low risk of the disease. Some patients, particularly those with high pre-treatment $\beta$ hCG levels, who are assigned to the LRGTN group and are thus administered with a single-agent chemotherapy, in fact, later, require therapy conversion. Although the disease is highly sensitive to chemotherapy [3], early diagnosis and the best choice of treatment regimen are important for patients; and this may also shorten the treatment time, and consequently, its costs. If misdiagnosed at an early stage, malignant gestational trophoblastic disease may metastasize to distant organs. In addition, early diagnosis can permit early application of the appropriate treatment, which can increase the patient's chances of a faster recovery. Correct diagnosis can be obtained effortlessly using analysis of the concentration of human chorionic gonadotropin. But selecting the right treatment for the patient is more complicated. The application of a therapy that is too gentle can trigger drug resistance, whereas choosing a too-aggressive therapy increases the incidence of unwanted side effects and a premature disqualification of the patient from the therapy. Either discontinuing or disturbing treatment may also cause drug resistance, with the consequence of the patient remaining unhealed, and eventually, patient mortality. Almost $99 \%$ of low-risk patients respond to monotherapy, whereas $70 \%$ of medium-risk patients (who scored 5 and 6 points) do not respond to the first-line treatment and require an adjustment of their treatment regimen. About $25-30 \%$ of patients with GTN develop resistance to the first-line chemotherapy and require alteration of their therapy [3]. One of the most significant steps in the course of a GTN resistant therapy is choosing the right moment to apply the triple or multi-agent regimen. Until now, diagnosis of resistance to single agent treatment with MTX has been based on the rise or stagnation of serum $\beta$ hCG levels during treatment, and (or) development of new metastases; however, no consensus on a defining guideline regarding the diagnosis of MTX resistance has been established [4]. Also, initially high levels of serum $\beta$ hCG are a known risk factor of MTX treatment failure, though its dynamics during the treatment of MTX-sensitive and MTX-resistant patients still require some investigation. The aim of our study was to investigate whether initial differences in $\beta$ hCG levels between the first and second doses of methotrexate are predictive of a future need for conversion to a multi-agent EMA/CO regimen.

\section{MATERIAL AND METHODS}

\section{Patient Characteristics}

A total of 46 patients diagnosed with low-risk GTN were retrospectively analyzed. All patients had been treated between November 2000 and September 2016 in the Department of Gynecology, Obstetrics and Gynecologic Oncology of the Poznan University of Medical Sciences, which is the Polish Reference Center for GTD. All women were initially qualified for a 5-day, single-agent therapy based on the WHO criteria ( $25 \mathrm{mg}$ of Methotrexate once daily, administered intramuscularly). The pre-treatment evaluation of each patient included a physical examination, measuring the serum human chorionic gonadotropin levels, and conducting pelvic ultrasonography and chest radiography. Serum $\beta$ hCG levels were evaluated before each chemotherapy cycle. Treatment was stopped if the $\beta$ hCG levels normalized ( $\leq 1 \mathrm{mIU} / \mathrm{mL}$ ) (MTX group). If there was an increase, a plateauing or a decrease of $\beta$ hCG concentrations of less than $15 \%$ between any of the previous and following courses of MTX, the therapy was converted to a multi-agent regimen. At some point, 22 patients were considered resistant to the treatment of the 5-day MTX therapy and were subsequently treated with an EMA/CO regimen (EMA/CO group).

The study was conducted in accordance with the requirements of the Declaration of Helsinki and the study protocol was approved by the Ethical Review Board of the Poznan University of Medical Sciences, Poland (Decision No. 425/14).

\section{Statistical analysis}

Analysis of data was performed in R programming language (version 3.4.1) using RStudio (version 0.98.1060). The normality of the distribution was verified using the Shapiro test. Groups with a normal distribution were compared using the t-Student test. Groups with distributions that deviated from normal were compared using the Mann-Whitney test. The results were considered significant for $p$ values $<0.05$. $P$ values are specified in the figures included in this paper. Plots were generated using ggplot2 and ggpubr $R$ packages. $P$ values were calculated 
and added to the plots using the stat_compare_means() function. Boxplots were generated using the geom boxplot() function. Plot whiskers extend to the most extreme data point, within 1.5 times the interquartile range of the box. The upper and lower "hinges" correspond to the first and third quartiles. The central line in the boxplot represents the median. Dots in the plot were generated using the function geom_jitter() and correspond to the single measurements of individual patients. An R package plotROC was applied to produce receiver operating characteristics (ROC) curves and to calculate the area under the curve (AUC). The cutoff values were determined using the OptimalCutpoints R package based on the Youden index. The predictive performance of the markers were compared using the Delong test and were computed using the roc.test() function from the pROC R package.

\section{RESULTS}

The range of initial $\beta$ hCG concentrations in the EMA/CO group members equaled $21.53 \mathrm{mIU} / \mathrm{mL}-192.610 .0 \mathrm{mIU} / \mathrm{mL}$.
Before conversion to the EMA/CO therapy, patients received between 200 and 5050mg of MTX (between 1 and 16 methotrexate cycles were administered, with patients receiving $1,2,3,4,5,7,8$ or 16 MTX courses). In the MTX group, 24 patients received between 3 and 10 administrations of methotrexate (the range of initial $\beta$ hCG: $56.5 \mathrm{mlU} / \mathrm{mL}-$ $360.397 .0 \mathrm{mlU} / \mathrm{mL}$ ) and were successfully managed using MTX therapy (with an overall MTX dose of 375-900 mg) without further conversion to an EMA/CO regimen (MTX group).

We observed that the median of the initial $\beta$ hCG levels before the onset of methotrexate therapy was higher in the EMA/CO (median = 6275) group than in the MTX (median $=532)$ group $(p=0.034$, Mann-Whitney test, Fig. 1). To analyze the initial dynamics of $\beta$ hCG, we plotted the corresponding concentrations at the first and second doses of methotrexate (Fig. 2A). To compare the decrease in $\beta$ hCG levels between patients, we plotted the percentage change relative to the initial level at dose 1 of methotrexate (Fig. 2B). We observed that the average drop (the dashed line in Figure 2B) in the relative $\beta$ hCG values was more pronounced in the MTX

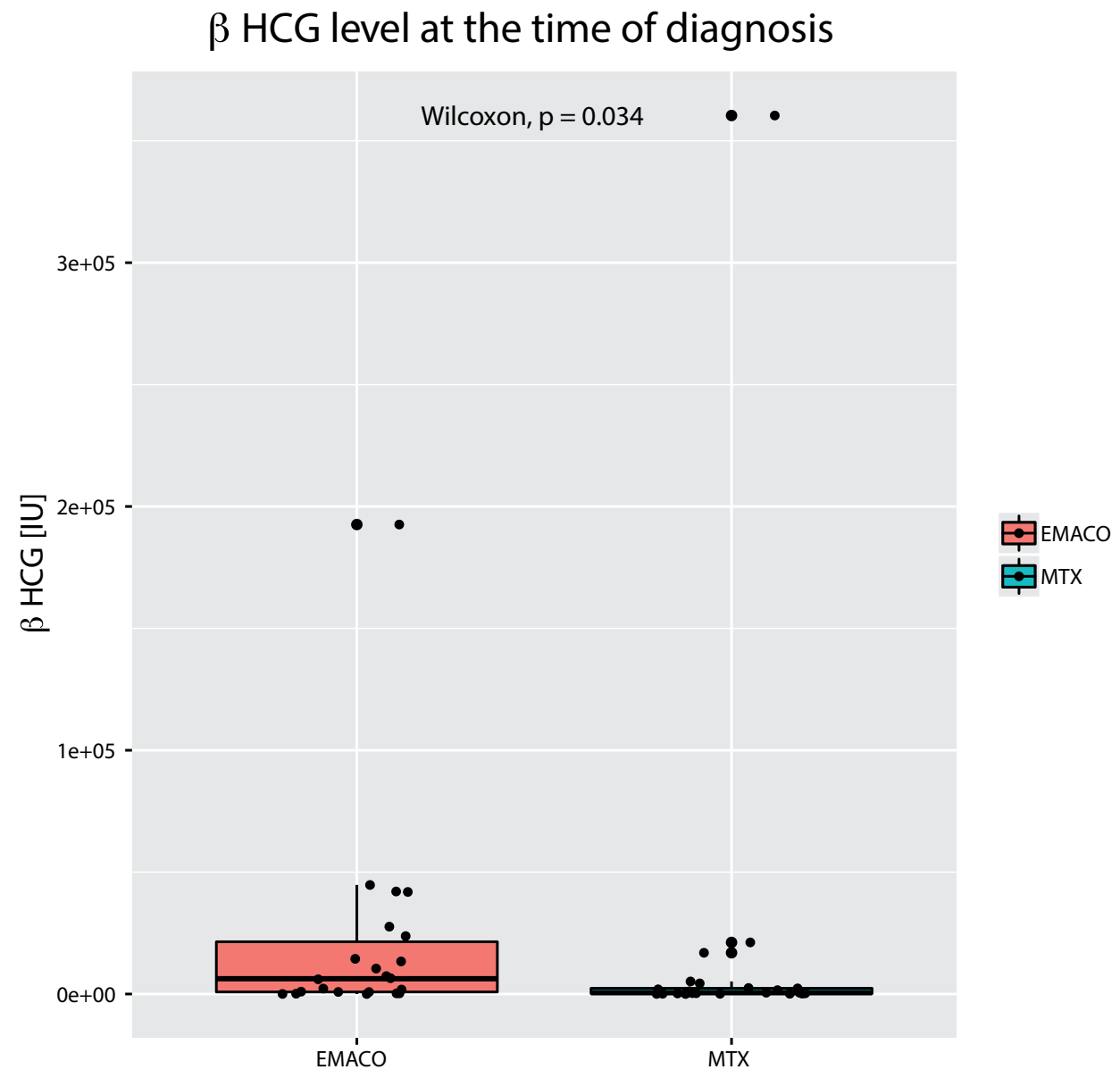

Future chemotherapy regimen

Figure 1. $\beta$ hCG level at the time of diagnosis 
A

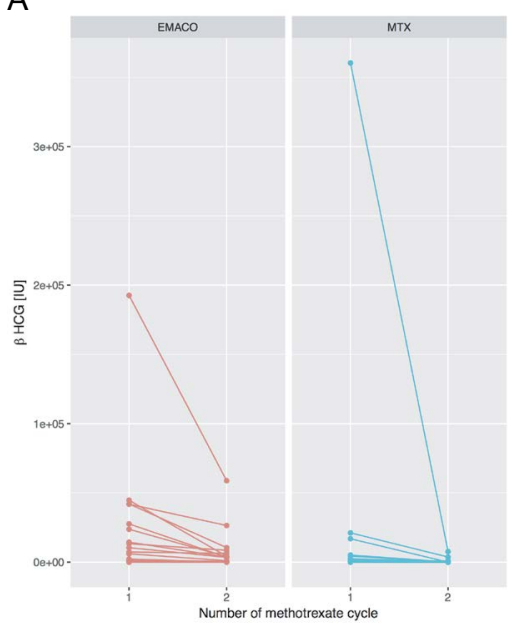

B

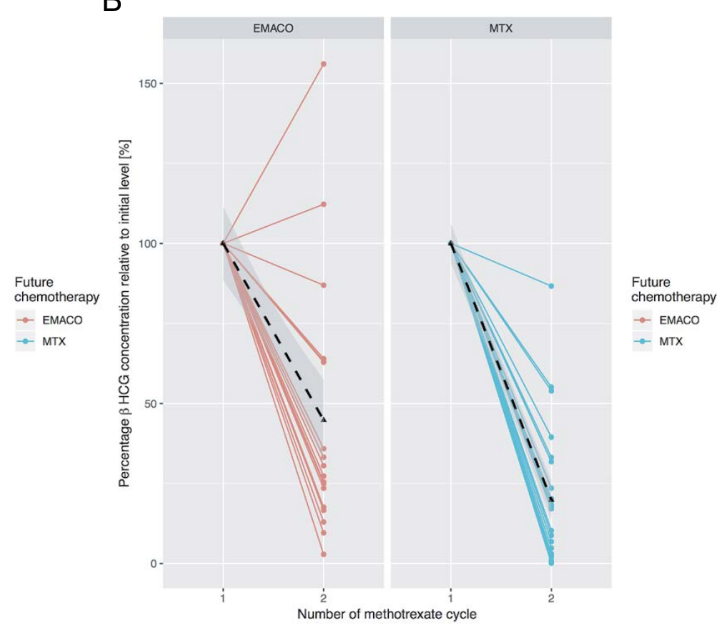

Figure 2. A. Concentrations of $\beta$ hCG at the first and second doses of methotrexate $\mathbf{B}$. The percentage change relative to the initial level at dose 1 of methotrexate

group. To determine whether that difference was relevant, we compared the percentage change in the $\beta$ hCG levels between the EMA/CO and MTX groups. We demonstrated that the gradient in the relative $\beta$ hCG values was more negative in the MTX (median decrease $=82.7 \%$, range: $13.3-99.9 \%$ ) group than in the EMA/CO (median decrease $=71.1 \%$, range: from an increase of $56.1 \%$ to a decrease of $97.1 \%$ ) group $(p=0.0079$, Mann-Whitney test, Fig. 3). To evaluate the predictive value of those parameters we determined their specificity and sensitivity in predicting the future need for conversion to an EMA/CO regimen (Fig. 4A and 4B). An analysis of the ROC curves implied optimal cutoff values for the initial $\beta$ hCG (6054 IU, sensitivity $=55 \%$, specificity $=86 \%$, based on Youden index) and the percentage change in the $\beta$ hCG levels (a decrease of $76.5 \%$, sensitivity $=72 \%$, specificity $=71 \%$, based on Youden index). The parameters of measuring the initial $\beta$ hCG levels in comparison with the percentage changes in $\beta$ hCG levels, and then comparing these results between the EMA/CO and MTX groups, showed that their predictive value did not differ (AUC for the initial $\beta$ hCG and the percentage change in the $\beta$ hCG levels were 0.68 and 0.74 , respectively; DeLong test, $p=0.96$ ). Altogether, we have shown that patients who will, in the future, be resistant to methotrexate and who will require an EMA/CO therapy (EMA/CO group) have a higher initial value of $\beta$ hCG and a lower drop in the percentage of the $\beta$ hCG value when measured between the first and second administrations of methotrexate.

\section{DISCUSSION}

Both defining and treating low-risk GTN remain somewhat controversial [4]. Tumor resistance is described as an increase in hCG levels or their stagnation over a 2 to 3-week period [5], although there is no internationally accepted consensus on the definition of drug resistance resulting from chemotherapy [6]. There is also no diagnostic tool available to predict chemoresistance and consequently to establish an appropriate treatment schema at the beginning of therapy.

In our study, we focused on only one factor (the regression of $\beta$ hCG levels between the first and second doses of methotrexate) as the prognostic indicator of methotrexate resistance. We aimed to evaluate whether the patients, who would need converting to a single-agent therapy in the future, have a lower decrease in their $\beta$ hCG levels between the first and second doses of MTX administered for LRGTN. MTX was the first agent used and is still the most common drug used in the treatment of LRGTN [7]. It has already been shown that a 5-day schema has minimal toxicity, and this compares favorably with the findings of other reports using similar methotrexate protocols [8-11]. Therefore, we used a 5-day regimen of methotrexate as the initial treatment for low-risk GTN. We noticed that the median initial $\beta$ hCG level prior to the onset of methotrexate therapy was higher in the EMA/CO group than in the MTX group. Our research also revealed that patients who would become resistant to methotrexate in the future, and therefore require EMA/CO therapy, have a lower percentage decrease in their $\beta$ hCG values when measured between the first and second administrations of methotrexate.

Several research projects on trophoblastic disease have concentrated on factors associated with hCG levels before. In one large study, the authors described the regression of serum hCG levels in patients with low-risk GTN who were successfully treated with MTX. The same authors revealed that serum $\mathrm{hCG}$ levels preceding the fourth and sixth single-agent chemotherapy courses proved to have excellent diagnostic 


\section{$\beta$ HCG change between cycle 1 and 2 of methotrexate therapy}

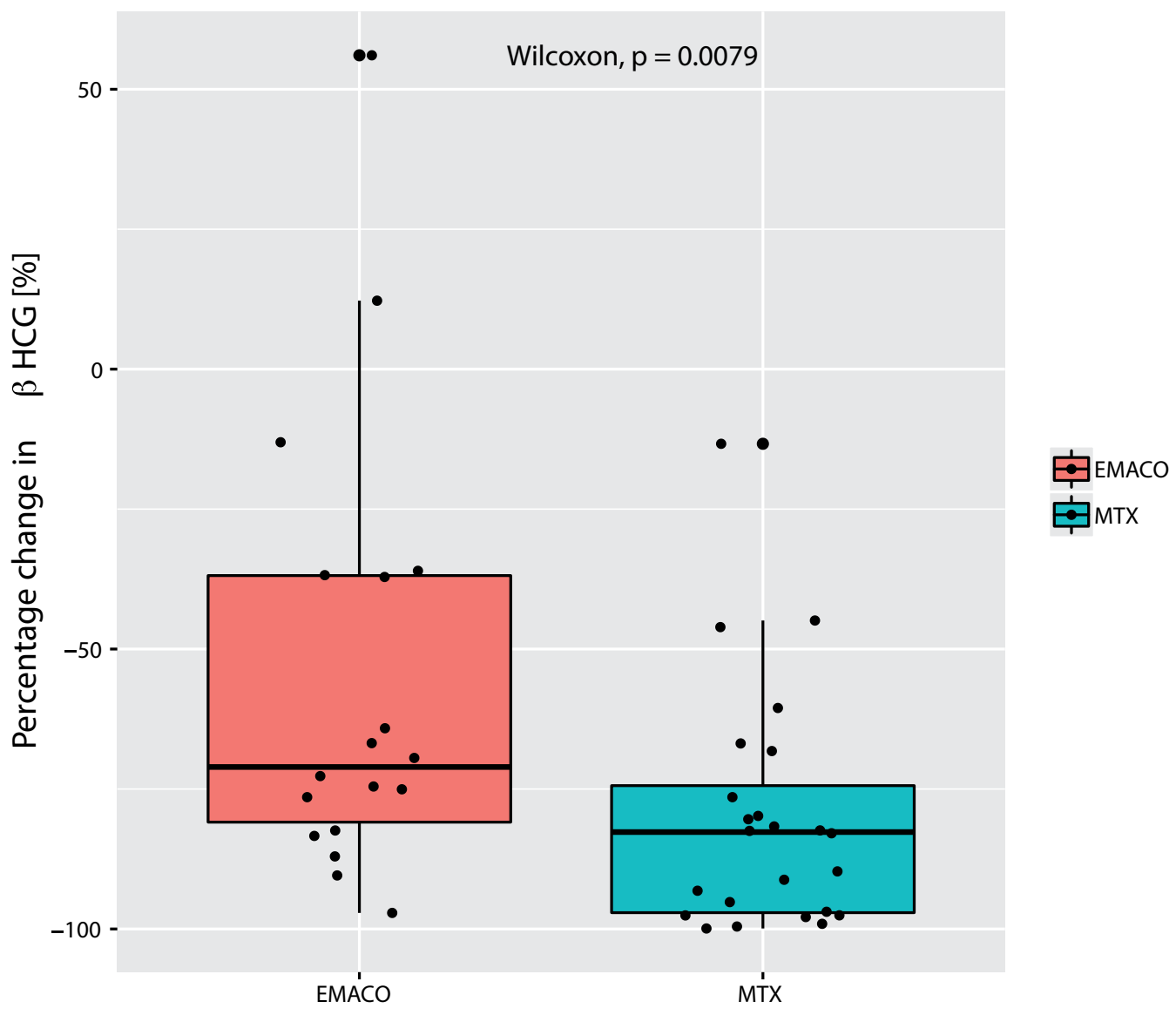

Future chemotherapy regimen

Figure 3. $\beta$ hCG change between dose 1 and 2 of methotrexate therapy
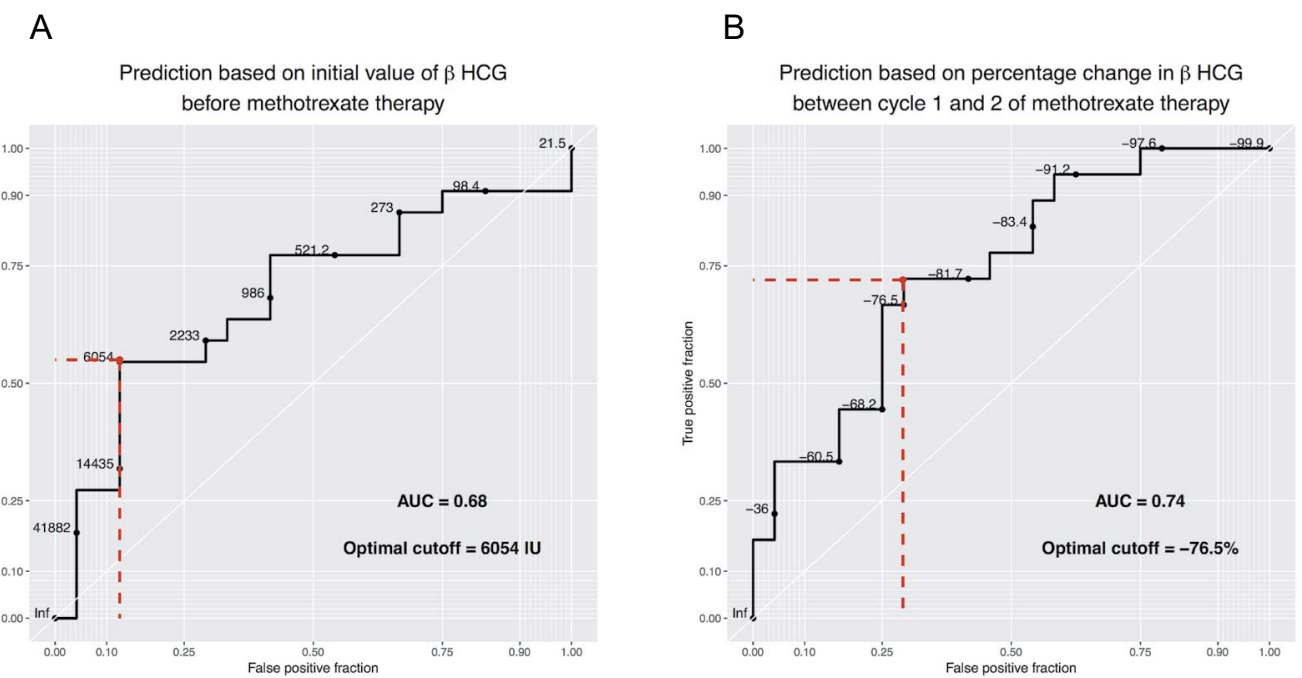

Figure 4. A. Prediction based on initial value of $\beta$ hCG before methotrexate therapy B. Prediction based on percentage change in $\beta$ hCG between cycle 1 and 2 of methotrexate therapy 
accuracy for identifying resistance to single-agent chemotherapy, with an area under the curve (AUC) of 0.949 and 0.975 , respectively, in the ROC curve analysis [12].

Maesta et al. [4] examined the time taken to achieve hCG remission in patients with low-risk postmolar GTN. The authors confirmed that a complete mole histology prior to GTN, the presence of metastatic disease, the use of a multi-agent therapy and a higher FIGO score were all independent factors associated with a longer time to hCG remission in patients with low-risk GTN. Chapman-Davis et al. [8] looked for the factors connected with resistance to single-agent methotrexate chemotherapy. Those authors described the presence of metastases, a clinicopathologic diagnosis of choriocarcinoma, higher FIGO/WHO scores and higher pretreatment hCG levels as factors associated with resistance to initial chemotherapy. In another research project, it was revealed that patients diagnosed with LRGTN and with a pre-treatment $\mathrm{hCG}>400.000 \mathrm{IU} / \mathrm{L}$ should receive multi-agent chemotherapy (EMA/CO) from the onset of treatment due to a poor response to a methotrexate-folinic acid (MTX-FA) regimen [13]. Also, Soper et al [14], reported the influence of high pre-treatment hCG levels on a negative response for drug resistance, and this finding coincides with our own results.

You et al. [15-17] concentrated on finding a model for hCG that may be useful in predicting MTX resistance. Model-building is demanding and challenging, but the authors' results may have practical clinical application [17]. The NRG Oncology/Gynecologic Oncology Group-174 (GOG-174) trial showed the value of modelling the kinetic parameters of hCG decline regarding the resistance of the disease to a single-agent chemotherapy; and in fact, their results revealed that their modelling could predict the individual risk of such resistance [15]. In an earlier study, the same authors confirmed that it is possible to design an individual model for hCG decline curves using a kinetic population approach with LRGTN patients, and that derived parameters were strong early predictors of MTX resistance [16]. In their subsequent research, the same authors confirmed the superior predictive power of the kinetic population modelling approach. The authors showed that by using individually calculated modelling of the residual production of hCGres values, it was possible to accurately identify about $80 \%$ of the patients who would develop MTX resistance [17].

Further retrospective research based on a larger population of patients is desired to give results that will enable early and adequate treatment for patients with low-risk GTN. Identifying the prognostic factors associated with effective treatment choices may help avoid unnecessary prolongation of the therapy and therefore minimize the side effects of chemotherapy and treatment costs and reduce patient anxiety.

\section{CONCLUSIONS}

1. Women with initially higher $\beta$ hCG levels have a greater risk of developing MTX chemo resistance.

2. It would be advantageous to consider administering an EMA/CO regimen in women with LRGTN when their initial $\beta$ hCG levels are greater than 6000 .

\section{Author Contributions}

Paulina Banach and Ewa Nowak-Markwitz designed the research; Paulina Banach and Ewa Nowak-Markwitz contributed important samples; Mikolaj Piotr Zaborowski performed the research; Paulina Banach, Natalia Izycka and Anna Romala collected data; Paulina Banach, Mikolaj Piotr Zaborowski and Ewa Nowak-Markwitz analyzed data; Paulina Banach, Mikolaj Piotr Zaborowski, Natalia Izycka, Anna Romala and Ewa Nowak-Markwitz wrote the paper; and all authors have read and approved the submitted manuscript.

\section{Conflicts of Interest}

The authors all declare they have no conflicts of interest.

\section{REFERENCES}

1. Uberti EM, Fajardo Md, da Cunha AG, et al. Treatment of low-risk gestational trophoblastic neoplasia comparing biweekly eight-day Methotrexate with folinic acid versus bolus-dose Actinomycin-D, among Brazilian women. Rev Bras Ginecol Obstet. 2015; 37(6): 258-265, doi: 10.1590/SO100-720320150005366, indexed in Pubmed: 26200823.

2. FIGO staging for gestational trophoblastic neoplasia 2000. International Journal of Gynecology \& Obstetrics. 2002; 77(3): 285-287, doi: 10.1016/s0020-7292(02)00063-2.

3. Aminimoghaddam S, Nezhadisalami F, Anjidani S, et al. Outcome of treatment with EMA/EP (etoposide methotrexate and actinomycin-D/ etoposide and cisplatin) regimen in gestational trophoblastic neoplasia. Med J Islam Repub Iran. 2018; 32: 36, doi: 10.14196/mjiri.32.36, indexed in Pubmed: 30159287.

4. Maestá I, Growdon WB, Goldstein DP, et al. Prognostic factors associated with time to hCG remission in patients with low-risk postmolar gestational trophoblastic neoplasia. Gynecol Oncol. 2013; 130(2): 312-316, doi: 10.1016/j.ygyno.2013.05.017, indexed in Pubmed: 23707672.

5. Foulmann K, Guastalla JP, Caminet N, et al. What is the best protocol of single-agent methotrexate chemotherapy in nonmetastatic or low-risk metastatic gestational trophoblastic tumors? A review of the evidence. Gynecol Oncol. 2006; 102(1): 103-110, doi: 10.1016/j.ygyno.2006.02.038, indexed in Pubmed: 16624388.

6. Kanno T, Matsui H, Akizawa Y, et al. Treatment results of the second-line chemotherapy regimen for patients with low-risk gestational trophoblastic neoplasia treated with 5-day methotrexate and 5-day etoposide. J Gynecol Oncol. 2018; 29(6): e89, doi: 10.3802/jgo.2018.29.e89, indexed in Pubmed: 30207097.

7. Gilani MM, Fariba B, Behtash N, et al. The WHO score predicts treatment outcome in low risk gestational trophoblastic neoplasia patients treated with weekly intramuscular methotrexate. J Cancer Res Ther. 2013; 9(1): 38-43, doi: 10.4103/0973-1482.110357, indexed in Pubmed: 23575072.

8. Chapman-Davis E, Hoekstra AV, Rademaker AW, et al. Treatment of nonmetastatic and metastatic low-risk gestational trophoblastic neoplasia: factors associated with resistance to single-agent methotrexate chemotherapy. Gynecol Oncol. 2012; 125(3): 572-575, doi: 10.1016/j. ygyno.2012.03.039, indexed in Pubmed: 22449733.

9. Hammond CB, Hertz R, Ross GT, et al. Primary chemotherapy for nonmetastatic gestational trophoblastic neoplasms. Am J Obstet Gynecol. 1967; 98(1): 71-78, indexed in Pubmed: 4290287.

10. Hammond CB, Soper JT, Weed JC, et al. Treatment of metastatic trophoblastic disease: good and poor prognosis. Am J Obstet Gynecol. 1973; 115(4): 451-457, indexed in Pubmed: 4346614. 
11. Soper JT, Clarke-Pearson DL, Berchuck A, et al. 5-day methotrexate for women with metastatic gestational trophoblastic disease. Gynecol Oncol. 1994; 54(1): 76-79, doi: 10.1006/gyno.1994.1169, indexed in Pubmed: 8020843.

12. van Trommel NE, Massuger LF, Schijf CP, et al. Early identification of resistance to first-line single-agent methotrexate in patients with persistent trophoblastic disease. J Clin Oncol. 2006; 24(1): 52-58, doi: 10.1200/JCO.2005.03.3043, indexed in Pubmed: 16382113.

13. McGrath S, Short D, Harvey R, et al. The management and outcome of women with post-hydatidiform mole,low-risk'gestational trophoblastic neoplasia, but hCG levels in excess of 100000 IU I(-1). Br J Cancer. 2010; 102(5): 810-814, doi: 10.1038/sj.bjc.6605529, indexed in Pubmed: 20160727.

14. Soper JT, Clarke-Pearson DL, BerchuckA, et al. 5-day methotrexate for women with metastatic gestational trophoblastic disease. Gynecol Oncol. 1994; 54(1): 76-79, doi: 10.1006/gyno.1994.1169, indexed in Pubmed: 8020843.
15. You B, Deng W, Hénin E, et al. Validation of the Predictive Value of Modeled Human Chorionic Gonadotrophin Residual Production in Low-Risk Gestational Trophoblastic Neoplasia Patients Treated in NRG Oncology/Gynecologic Oncology Group-174 Phase IIITrial. Int J Gynecol Cancer. 2016; 26(1): 208-215, doi: 10.1097/IGC.0000000000000581, indexed in Pubmed: 26569059.

16. You B, Pollet-Villard M, Fronton L, et al. Predictive values of hCG clearance for risk of methotrexate resistance in low-risk gestational trophoblastic neoplasias. Ann Oncol. 2010; 21(8): 1643-1650, doi: 10.1093/annonc/mdq033, indexed in Pubmed: 20154304.

17. You B, Harvey R, Henin E, et al. Early prediction of treatment resistance in low-risk gestational trophoblastic neoplasia using population kinetic modelling of hCG measurements. Br J Cancer. 2013; 108(9): 1810-1816, doi: 10.1038/bjc.2013.123, indexed in Pubmed: 23591194. 